\title{
Generation of interleukin-13 receptor alpha2 antigen expressing modified vaccinia ankara recombinant virus for potential cancer immunotherapy
}

\author{
Yuki Sato, Ramjay Vatsan, Bharat H Joshi, Syed R Husain, Raj K Puri \\ From Society for Immunotherapy of Cancer 29th Annual Meeting \\ National Harbor, MD, USA. 6-9 November 2014
}

Genetically modified recombinant poxviruses have shown promise in preclinical models of cancer immunotherapy due to their ability to induce effective cell-mediated immunity against target tumor-associated antigens (TAA). One such vector, recombinant Modified Vaccinia Ankara (MVA), is capable of expressing foreign genes in infected host cells. MVA is replication restricted in most mammalian cells exemplifying a unique safety profile. We have demonstrated that the interleukin-13 receptor $\alpha 2$ (IL-13R $\alpha 2)$ is selectively expressed in various solid tumors but not in normal tissues making it a promising TAA. Prophylactic and therapeutic vaccination with a plasmid vector expressing IL-13R $\alpha 2$ caused only partial regression of established tumors [1], suggesting that host immune responses against IL-13R $\alpha 2$ needed further enhancement. Thus, we constructed a recombinant MVA (rMVA-IL13R $\alpha 2$ ) expressing both IL-13R $\alpha 2$ and a green fluorescent protein (GFP) reporter gene. Purified virus titration by immunostaining using anti-vaccinia antibody and anti-IL-13R $\alpha 2$ antibody confirmed the identity and purity of the recombinant MVA. Western Blot analysis showed the presence of IL-13R $\alpha 2$ protein $(65 \mathrm{kDa})$. Flow cytometric analysis of IL-13R $\alpha 2$ negative T98G glioma cells infected with rMVA-IL13R $\alpha 2$ virus (T98G-IL13R $\alpha 2$ ) demonstrated surface expression of IL-13R $\alpha 2$, indicating the infectivity potential of the recombinant virus. Incubation of T98G-IL13R $\alpha 2$ cells with varying concentrations (0-100 ng/ml) of IL13-PE (interleukin-13 fused to truncated Pseudomonas exotoxin [2] resulted in depletion of $\mathrm{GFP}^{+}$ T98G-IL13R $\alpha 2$ cells in a concentration-dependent manner.
Higher concentrations of IL13-PE (10-1000 ng/ml) also inhibited the protein synthesis in T98G-IL13R $\alpha 2$ compared to cells infected with control pLW44-MVA. We further observed that IL13-PE treatment of rMVA-IL13R $\alpha 2$ infected chicken fibroblast, DF-1 cells led to a reduction in virus titer compared to untreated cells. These results indicate that rMVA-IL13R $\alpha 2$ virus can successfully infect mammalian cells and express IL-13R $\alpha 2$ in a biologically active form on the cell surface. The immunization studies of rMVA-IL13R $\alpha 2$ are ongoing in a syngeneic mouse model of metastatic breast carcinoma. Based on in vitro results, we expect the rMVA-IL13R $\alpha 2$ to be a useful agent in tumor immunotherapy as a vaccine alone and in combination with other therapeutic agents to eradicate metastatic tumors.

Published: 6 November 2014

\section{References \\ 1. Nakashima $H$, Terabe $M$, Husain SR, Puri RK: A novel combination immunotherapy for cancer by IL-13Ra2-targeted DNA vaccine and immunotoxin in murine tumor models. J Immunol 2011, 187:4935-4946. \\ 2. Husain SR, Puri RK: Interleukin-13 receptor-directed cytotoxin for malignant glioma therapy: from bench to bedside. J Neuro-Oncol 2003, 65:37-48.}

doi:10.1186/2051-1426-2-S3-P58

Cite this article as: Sato et al:: Generation of interleukin-13 receptor alpha2 antigen expressing modified vaccinia ankara recombinant virus for potential cancer immunotherapy. Journal for ImmunoTherapy of Cancer 2014 2(Suppl 3):P58. 